\title{
Adrenal insufficiency resulting from immunotherapy: a rare but life- threatening side effect
}

\author{
Muhammad Yusuf Shaharudin*, Mohammad Zulkhairi
}

Department of Internal Medicine, Geriatrics and Palliative Unit, Raja Isteri Pengiran Anak Saleha (RIPAS) Hospital, Brunei Darussalam

Received: 19 October 2021

Revised: 18 November 2021

Accepted: 19 November 2021

\section{*Correspondence:}

Dr. Muhammad Yusuf Shaharudin,

Email: usopan@gmail.com

Copyright: (C) the author(s), publisher and licensee Medip Academy. This is an open-access article distributed under the terms of the Creative Commons Attribution Non-Commercial License, which permits unrestricted non-commercial use, distribution, and reproduction in any medium, provided the original work is properly cited.

\begin{abstract}
A 73-year-old male whom suffers from stage 4 adeno-squamous lung carcinoma with high programmed death-ligand 1 (PD-L1 immunohistochemistry-tumor proportion score (TPS) $>50$ began his treatment with a type of immune checkpoint inhibitors (ICI) therapy. After $8^{\text {th }}$ cycle of treatment, patient experiences vague symptoms of fever, lethargy and drowsy. Initial working diagnosis was infection and empirical treatment was initiated. Subsequently as patient's condition did not improve despite appropriate antibiotics, other diagnosis was considered which include adrenal insufficiency and this confirms after checking cortisol level. Hydrocortisone was started promptly and patient clinical condition improved. Decision was to stop ICI treatment interim. Consideration to re-challenge again with ICI may be considered once patient is more fit in the future. Adrenal insufficiency following ICI treatments are rare and misdiagnosed due to its non-specific symptoms. Nonetheless, it comes with high morbidity and mortality. Hence, we wanted to emphasize more on this condition as more elderly patients whom suffers from various type of cancers will be exposed towards ICI treatment.
\end{abstract}

Keywords: Immunotherapy, Adrenal insufficiency, Immune-checkpoint inhibitor, Pembrolizumab, Adrenal insufficiency

\section{INTRODUCTION}

Many patients suffer from various types of cancers. The introduction of ICI therapy in recent years have revolutionalised cancer treatment as prognosis of several tumor types for example renal cell carcinoma, non-small cell lung carcinoma, and skin melanoma, to name a few has improved. ${ }^{1-3}$ This would mean more elderly will be exposed and treated with ICI. However, ICI may lead to trigger of immune related adverse events (irAEs).

Adrenal insufficiency is being reported been one of the irAEs however the incidences are low and for primary adrenal insufficiency especially are thought to be extremely rare of only $0.7 \%$ being reported in randomized clinical trials. ${ }^{4}$ This is however thought to be underdiagnosed despite significant morbidity and mortality with death of $7.3 \%$ associated to it. ${ }^{5,6}$ Hence it is important to recognize the condition early and treated appropriately.

\section{CASE REPORT}

We presented a case of 73 years old male with ECOG (Eastern co-operative oncology group) performance status of 1 with medical history of type 2 diabetes, hypertension, hyperlipidemia, ischemic heart disease and has been a chronic smoker for 40 years. He was diagnosed with stage 4 adenosquamous lung carcinoma in February 2020 with positive programmed death-ligand 1 (PD-L1) immunohistochemistry- tumor proportion score (TPS) of 95\%. Initial positron emission tomography (PET) scan showed large mass at right lower lobe with hypermetabolic 
metastatic right hilar, subcarinal lymph nodes and right pelvis. No FDG avid adrenal metastasis.

As molecular investigations and PDL1 TPS score were sent abroad, he was initially started with first cycle of chemotherapy with carbo/taxol every week for 3 weeks in March 2020. After the result of his PD-L1 expression was released, his treatment was switched to pembrolizumab, a type of ICI-therapy, $200 \mathrm{mg}$ fixed dose every 3 weeks starting from $2^{\text {nd }}$ April 2020. His treatment was interrupted in mid-June because of evaluation of mild acute kidney injury with poorly controlled blood pressure. Ultrasound revealed grade 1 cortico-medullary changes and no hydronephrosis. Echocardiography showed mild left ventricular hypertrophy with ejection fraction $(\mathrm{EF})$ of $66 \%$. His treatment was subsequently resumed back until cycle 8 on 22/9/2020.

Following the $8^{\text {th }}$ cycle of pembrolizumab, patient started to develop severe asthenia, anorexia with nausea and vomiting. He presented to emergency department on 27/9/2020 with fever, drowsy and vomiting. He is febrile on arrival, blood pressure was still within normal range; however, he has mild hypoglycemia of $3.1 \mathrm{mmol} / \mathrm{L}$ (ref range: 4.0-7.9 $\mathrm{mmol} /$ ). His physical examinations were unremarkable.

His initial inflammatory markers were just mildly elevated with C-reactive protein (CRP) of $2.8 \mathrm{mg} / \mathrm{dL}$ (ref range: $0.0-0.4 \mathrm{mg} / \mathrm{dL}$ ) and procalcitonin of $0.8 \mathrm{ng} / \mathrm{ml}$ (ref range: $>2.0 \mathrm{ng} / \mathrm{ml}$ represent severe sepsis. $<0.5 \mathrm{ng} / \mathrm{ml}$ represent low risk). The rest of his initial blood test showed hyponatremia at $129 \mathrm{mmol} / \mathrm{L}$ (ref range: $136-145 \mathrm{mmol} / \mathrm{L}$ ) with potassium level of $4.9 \mathrm{mmol} / \mathrm{L}$ (ref range: 3.5-5.0 $\mathrm{mmol} / \mathrm{L}$ ) and acute renal failure, serum creatinine of 189.4 umol/L (ref range: 63-110 umol/L). Hyperesonophilia was not present. Chest X-ray did not show any new findings except for the known lung mass (Figure 1) and normal urinary dipstick. He was empirically treated with antibiotics but did not improve after 48 hours.

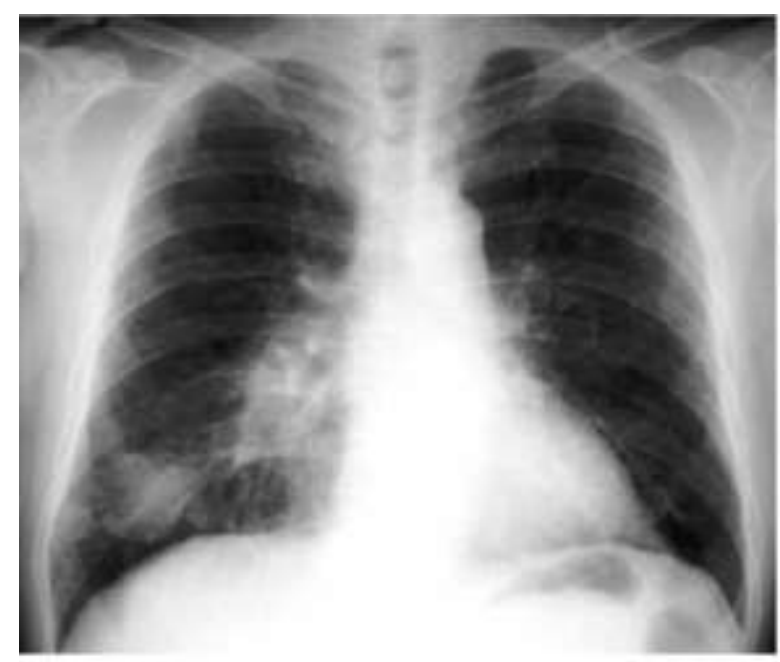

Figure 1: Chest x-ray on admission with the known stable lung mass.
The diagnosis of acute adrenal crisis was confirmed by measurement of very low morning serum cortisol at 38 $\mathrm{nmol} / \mathrm{L}$ (ref range: $172-497 \mathrm{nmol} / \mathrm{L}$ ), equivalent to 1.377 $\mu \mathrm{g} / \mathrm{dl}$ on day 3 of admission. Adrenocorticotropic hormone level was not performed at the beginning in view of laboratory technical issue. Treatment with intravenous hydrocortisone was urgently initiated, and the patient rapidly improved afterwards.

Other pituitary axes showed normal TSH and prolactin level with elevation of FSH and LH level at 34.2 IU/L (ref range:1.5-12.4 IU/L) and 20.5 IU/L (ref range: 1.7-8.6 IU/L) respectively. Testosterone and thyroid function test level otherwise within normal range. GH and IGF-1 unable to be performed in view of the-our local laboratory limitations.

A computed tomography (CT) scan of thorax, abdomen and pelvis was undertaken which did not show evidence of adrenalitis or adrenal mass. The lung mass of concern has shown $82 \%$ regression of volume in size from the date of first diagnosis following treatment. An endocrinologist was consulted and had commented that on top of the adrenal insufficiency, the FSH and LH level with normal testosterone level are in keeping with subclinical primary hypogonadism which requires no treatment.

Patient was discharged with oral hydrocortisone with complete resolution of his original symptoms. His level of cortisol improves to $227 \mathrm{nmol} / \mathrm{L}$ (ref range: 172-497 $\mathrm{nmol} / \mathrm{L}$ ) one week from the first initiation of hydrocortisone. He will continue being seen in our clinic to monitor his progress and observing tolerance and side effects to steroid. His subsequent oncological treatment has to be stopped interim and will be discussed again for treatment re-challenge later on.

\section{DISCUSSION}

Pembrolizumab is a type of ICI that has been approved to be used in various cancers. It works by binding and blocking on Programmed-cell death protein 1 (PD-1) receptor of lymphocytes. Many cancers make proteins such as PD-L1 that bind to PD-1, thus shutting down the ability of the body to kill the cancer on its own. ${ }^{7}$ Inhibiting PD-1 on the lymphocytes prevents it from binding to ligands that deactivate an immune response, allowing the immune system to target and destroy cancer cells. ${ }^{8}$ This process, however can also result in an unchecked immune response that manifests clinically in the form of numerous autoimmune-like/inflammatory adverse effects. Examples of such adverse effects include dermatologic, hepatic, gastrointestinal, endocrine (pituitary, adrenal), renal, and pulmonary toxicities. Most adverse effects associated with ICI are mild or moderate, and the most common toxicities are rash and diarrhea. However, less common and potentially severe toxicities can occur (Table 1). ${ }^{9}$ 
Table 1: Immune related adverse reaction. ${ }^{9}$

\begin{tabular}{|c|c|}
\hline S. no. & Variables \\
\hline \multirow[t]{4}{*}{1.} & Common $(>10 \%)$ \\
\hline & Dermatitis, pruritis \\
\hline & Fever, chills, fatigue \\
\hline & Diarrhea \\
\hline \multirow[t]{15}{*}{2.} & Rare $(<10 \%)$ \\
\hline & Colitis \\
\hline & Hepatitis/liver enzyme abnormalities \\
\hline & Pancreatitis \\
\hline & Pneumonitis \\
\hline & Diabetes type 1 \\
\hline & $\begin{array}{l}\text { Endocrinopathies: Hypophysitis, thyroiditis, } \\
\text { adrenal insufficiency }\end{array}$ \\
\hline & Nephritis \\
\hline & Myocarditis \\
\hline & Myelitis \\
\hline & Guillain-Barre syndrome \\
\hline & Encephalitis \\
\hline & $\begin{array}{l}\text { Hemolytic anemia and immune } \\
\text { thrombocytopenia }\end{array}$ \\
\hline & $\begin{array}{l}\text { Toxic epidermal necrolysis; Steven- Johnson } \\
\text { syndrome }\end{array}$ \\
\hline & Infusion reaction \\
\hline
\end{tabular}

Adrenal insufficiency is the inability of adrenal gland to produce sufficient number of glucocorticoids and/or mineral corticoids. These hormones are important to regulate our salt, fluid and energy, and can be life threatening if it did not work properly. In healthy individuals, the hypothalamic-pituitary axis will provide positive and negative feedback mechanisms in order to modulate a number of physiological processes such as immunity, fertility, and the body's response to stress. ${ }^{10}$ Disruption in hormone production at any level (pituitary or adrenal) leads to decreased cortisol production and hence leading to adrenal insufficiency.

Many common manifestations of adrenal insufficiency are vague, sometimes insidious and non-specific for example; fatigue, anorexia, hypoglycemia and hyponatremia, to name a few. This can be easily overlooked if adverse events of immunotherapy are not suspected. This is especially when adrenal insufficiency following ICI therapy incidence are very low at $0.7 \% .{ }^{4}$ although this may be under reported. In the case demonstrated above, the symptoms and sign on initial presentation may mislead clinician into treating the patient as infection. This is especially his blood pressure was within normal limits and the potassium still within normal range although at the upper limit of normal range. After further assessment, this adrenal crisis was the suspected as well as treated accordingly.

ICI- induced adrenal insufficiency can be due to central cause, which is a part of hypophysis or from primary adrenal insufficiency (PAI). Systemic reviews showed incidence in a range of $0.7-0.9 \% .^{4,6}$ Hypophysis its incidence however is at $3-6 \% .^{4}$ In the case that we presented, the patient likely to have PAI as the rest of pituitary axes were unremarkable.

Of all the ICI therapies, nivolumab accounted for $44.3 \%$ of cases of PAI, followed by ipilimumab at $23.6 \%$ and pembrolizumab at $11.7 \% .{ }^{6}$ Combination ICI therapy was thought to account around $17.9 \% .^{6}$ In terms of symptoms onset of developing into adrenal insufficiency, there were no clear define time frame. It can be within the first month of treatment, but there are cases being reported several years after starting treatment. Median time of 4 months were reported with large range from 6 days to 576 days. $^{6}$ In our case, the onset was about 5 months from the first initiation of pembrolizumab.

In a patient that is suspected to have ICI induced adrenal insufficiency, it is important to confirm with morning serum cortisol, ACTH and ACTH stimulation test. An early morning low serum cortisol concentration (less than $3 \mathrm{mcg} / \mathrm{dL}$ [80 $\mathrm{nmol} / \mathrm{L}]$ ) is strongly suggestive of adrenal insufficiency which is the case here. ${ }^{11}$

Mainstay treatment of adrenal insufficiency are with glucocorticoid and often with mineral corticoid. Stress dose corticosteroids should be immediately administered to any patient with suspected adrenal crisis. A typical stress dose of corticosteroids includes an initial dose of hydrocortisone $100 \mathrm{mg}$ IV followed by hydrocortisone 50 mg IV every 6 hours; this regimen can be subsequently tapered depending on the clinical course. ${ }^{12}$ For baseline doses of corticosteroids, glucocorticoid replacement with 15 to $25 \mathrm{mg}$ of hydrocortisone PO daily in divided doses provides adequate replacement for most patients with PAI. ${ }^{12}$ Mineralocorticoid replacement with fludrocortisone can be helpful in patients with confirmed aldosterone deficiency to reduce salt craving and postural hypotension and prevent hyponatremia and the hyperkalemia.

\section{CONCLUSION}

As more evidence seeing on the outcome of ICI therapy with many cancers, acute care and emergency departments will seing more sick patients exposed with ICI therapy. As with other treatments, adverse effects of ICI-therapy shall be familiarized by physicians attending so that care will be delivered appropriately. Adrenal insufficiency is one of the rare conditions related to ICI-therapy which comes with significant morbidity as well as the mortality associated with it. Early recognition and prompt treatment are important on dealing with this group of patients. The above case highlights the importance of being vigilant to the immune related side effect as the symptoms can be the non-specific.

\section{Funding: No funding sources Conflict of interest: None declared \\ Ethical approval: Not required}




\section{REFERENCES}

1. Motzer RJ, Tannir NM, Mc Dermott. Check Mate 214 Investigators. Nivolumab plus ipilimumab versus sunitinib in advanced renal cell carcinoma. $\mathrm{N}$ Eng $\mathrm{J}$ Med. 2018;378(14):1277-90.

2. Hellmann MD, Rizvi NA. Nivolumab plus ipilimumab as first line treatment for advanced nonsmall cell lung cancer (CheckMate 012); results of and open label phase 1; multicohort study. Lancet Oncol. 2017;18(1);31-41.

3. Larkin J, Chiarion- Sileni V. Combined nivolumab and ipilimumab or monotherapy in untreated melanoma. N Eng J Med. 2015;373(1):23-4.

4. Barroso-Sousa R, Barry WT, Garrido-Castro AC, Hodi FS, Min L, Krop IE, Tolaney SM, Incidence of Endocrine Dysfunction Following the Use of Different Immune Checkpoint Inhibitor Regimens: A Systematic Review and Meta-analysis. JAMA Oncol. 2018;4(2):173.

5. Brahmer JR, Lacchetti. Management of immunerelated adverse events in patients treated with immune checkpoint inhibitor therapy. American Society of Clinical Oncology Clinical Practice Guideline Summary. J Oncol Pract. 2018;14(4)247-9.

6. Grouthier V, Lebrun- Vignes B. Immune Checkpoint Inhibitor Associated Primary Adrenal Insufficiency; WHO VigiBase Report Analysis. Oncologist. 2020;25:696-701.

7. Syn Nicholas L, Teng Michele WL, Mok Tony SK, Soo Ross A. De-novo and acquired resistance to immune checkpoint targeting. Lancet Oncol. 2017;18(12):e731-41.

8. Pardoll DM. The blockade of immune checkpoints in cancer immunotherapy. Nature Reviews Cancer. 2014;12(4):252-64.

9. Champiat S, Lambotte O, Barreau E, Belkhir R, Berdelou A, Carbonnel $\mathrm{F}$ et al. Management of immune checkpoint blockade dysimmune toxicities: a collaborative position paper. Ann Oncol. 2016;27(4):559-74.

10. Sharon DeMorrow, Role of the HypothalamicPituitary-Adrenal Axis in Health and Disease, Int $\mathbf{J}$ Mol Sci. 2018;19(4):986.

11. Hagg E, Asplund K, Lithner. Value of basal plasma cortisol assays in the assessment of pituitary-adrenal insufficiency. Clin Endocrinology (Oxf) $.1987 ; 26(2): 221$.

12. Bornstein SR, Allolio B, Arlt W, Barthel A, DonWauchope A, Hammer GDm et al. Diagnosis and treatment of primary adrenal insufficiency: an Endocrine Society Clinical Practice Guideline. J Clin Endocrinol Metab. 2016;101(2):364-89.

Cite this article as: Shaharudin MY, Zulkhairi M. Adrenal insufficiency resulting from immunotherapy: a rare but life-threatening side effect. Int J Basic Clin Pharmacol 2021;10:1420-3. 